\title{
Aggregation induced emission in the tetraphenylthiophene crystal: The role of triplet states
}

\author{
Ljiljana Stojanović and Rachel Crespo-Otero* \\ School of Biological and Chemical Sciences, Queen Mary University of London, Mile End \\ Road, London, United Kingdom, E1 4 NS \\ E-mail: r.crespo-otero@qmul.ac.uk \\ Phone: +44(0)207882 8404
}

\begin{abstract}
Propeller-shaped molecules have received much attention due to their enhanced emission in the condensed phase (Aggregation Induced Emission, AIE) and their potential use in optoelectronic devices. In this contribution, we examine the excited state mechanisms of tetraphenyl-thiophene (TPT), one member of the family which features weaker AIE. We perform a detailed analysis of the potential energy surfaces with special focus on the role of triplet states considering the crystal structure, intermolecular interactions, exciton couplings and reorganisation energies in the vacuum and solid state. In contrast with other members of the propeller-shaped family, nonradiative decay in TPT is driven by bond breaking. Because of the significant spin-orbit couplings along the reaction coordinate, intersystem crossing plays an important role in the mechanism. Our calculations show that aggregation in the solid state hampers the access to internal conversion pathways, however, intersystem crossing is active in the crystal phase, which explains the weak AIE of this molecule. This new understanding of the role of triplet
\end{abstract}


states on the relaxation mechanisms of AIEgens has implications for the design of solid state highly-emissive materials based on TPT.
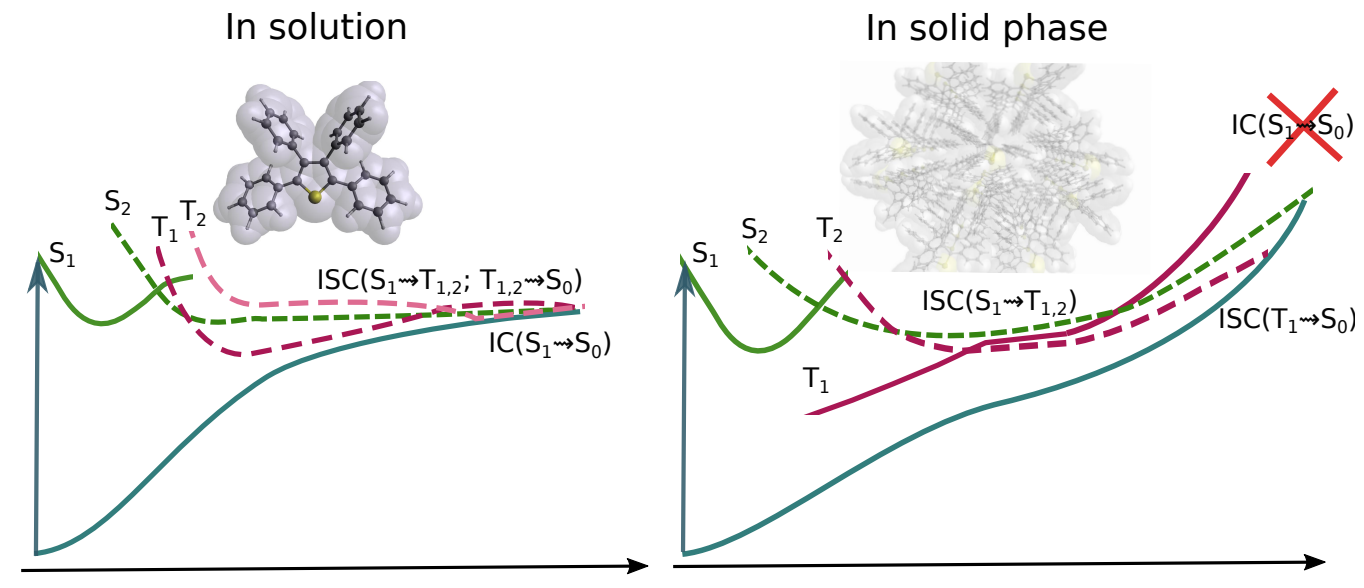


\section{Introduction}

One significant obstacle in the development of highly-emissive solid state organic materials is the decrease of emissive efficiency upon aggregation - aggregation caused quenching (ACQ). This phenomenon is common in planar $\pi$-conjugated organic fluorophores where $\pi-\pi$ stacking between monomers and other physical processes (e.g., energy transfer, inter- and intramolecular charge transfer) can severely hamper radiative emission. The development of materials with the opposite behaviour, i.e. aggregation induced emission (AIE), ${ }^{1}$ opened up new possibilities for applications in modern technologies, such as photonics, optoelectronic devices, biological probes, and fluorescent sensors. To highlight the interconnected effects of inter- and intramolecular interactions on the emission efficiency in different forms of solids, a more general term, solid state luminescence enhancement (SLE) has been proposed. ${ }^{2}$

Several approaches have been considered to explain the enhancement of emissive response in aggregate phases. One of the most popular models is the restriction of intramolecular modes (RIM), which considers the relevant role of low-frequency vibrations in nonradiative decay. ${ }^{3}$ The formalism developed by Shuai et al. based on Fermi's Golden Rule has shown that low-frequency motions are hampered in the solid state substantially decreasing nonradiative rates. ${ }^{4}$ This model is relevant in the weak electronic coupling regime, however if the molecule can explore regions of the potential energy surface characterised by large electronic couplings, the role of conical intersections as a driving force of nonradiative decay cannot be ignored. ${ }^{5}$ Blancafort et al. have proposed that suppression of nonradiative decay in crystal is induced by restricted accessibility of conical intersections (the RACI model). ${ }^{5-7}$

Significant efforts have been devoted to the investigation of silole-based propeller-shaped molecules which show an enhancement of fluorescence in the condensed phase, in contrast to the low quantum yields of their organic solutions. ${ }^{8,9}$ In the case of dimethyl-tetraphenylsilole (DMTPS) (Figure 1), the excited state relaxation in solution involves internal conversion through a ring-puckering conical intersection. ${ }^{7}$ This conical intersection becomes unaccessible in the solid state leading to emission. A similar relaxation mechanism has been re- 
cently used to explain the AIE in a cyclopentadiene-based propeller-shaped molecule $(1,2,3,4$ tetraphenyl-1,3-cyclopentadiene, TPC). ${ }^{10}$

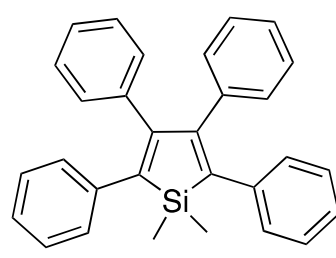

DMTPS

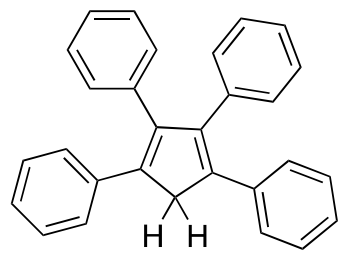

TPC

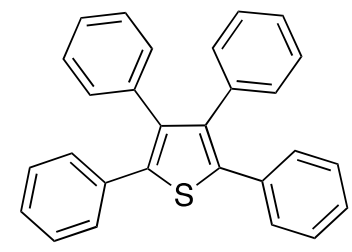

TPT

Figure 1: Structures of propeller-shaped molecules exhibiting aggregation induced emission enhancement.

Based on these examples, it is expected that other propeller-shaped molecules obtained by hetero-atom substitutions (for instance, Sulphur in the central unit, TPT, Figure 1) would also be promising AIEgens. ${ }^{11,12}$ Nie et al. have recently investigated luminescence properties of TPT in organic solution (THF) and in spin-coated films. ${ }^{12}$ According to their experiments, TPT is characterised by a weak aggregation-induced emission; the fluorescence quantum yield in solution is negligible $\left(\Phi_{r} \sim 0.023\right)$ only slightly increasing upon addition of water (at $80 \%$ volume fraction of water $\Phi_{r} \sim 0.06$ ). It has recently been found that TPT crystal features piezoresponsive luminescence, i.e. the emissive response of the crystal is enhanced within a certain range of applied pressure. ${ }^{13}$ This phenomenon has been attributed to the formation of new intermolecular interactions that restrict the rotation of phenyl rings, inhibiting the nonradiative mechanisms.

Various experimental strategies have been implemented to improve the emissive response of TPT derivatives in the condensed phase. For example, the design of bulky substituents that inhibit nonradiative decay, such as tetraphenyl-ethylene and alkoxy-chains, resulted in derivatives with high fluorescence quantum yield in the solid state. ${ }^{14,15}$ A number of AIEactive polymer structures based on TPT can also feature highly emissive properties. ${ }^{16-18}$ Despite the previous research, there is not a clear understanding of the excited state mech- 
anisms in TPT. Having in mind the gradual weakening of solid-state luminescence going from DMTPS, via TPC, to TPT (Figure 1), an interesting question arises: How does the substitution of a Silicon or Carbon atom by Sulphur alter the solid-state luminescence of propeller-shaped molecules?

To answer this question, we investigate the main radiative and nonradiative decay mechanisms of TPT in the vacuum and solid state taking into account singlet and triplet states. We consider crossings between states of different multiplicities, the effect of exciton couplings and intermolecular interactions on the main excited state mechanisms. Our calculations show that in contrast with previously studied propeller-shaped systems (DMTPS ${ }^{7}$ and $\mathbf{T P C}^{10}$ ), triplet states have a significant role on the deactivation of TPT, providing a plausible explanation for their photochemistry mechanism in solution and crystal phases.

\section{Computational Details}

We explored the excited state potential energy surfaces of TPT in vacuum, solvent and the solid state using single and multiconfigurational methods including time-dependent density functional theory (TD-DFT), resolution-of-the-identity coupled-cluster with approximate second-order excitations (RI-CC2), ${ }^{19-22}$ and complete active space perturbation theory (CASPT2) methods. ${ }^{23-25}$ We considered excited state minima and crossings involving singlet and triplet states.

We first assessed the performance of these electronic structure methods for the prediction of the absorption and emission energies. The ground state (FC), $\mathrm{S}_{1}$, and $\mathrm{T}_{1}$ minima were optimised at $\omega$ B97X-D/6-31G(d) ${ }^{26}$ and B3LYP/6-31G(d) levels of theory within the (TD-)DFT framework. ${ }^{27-31}$ The effect of the solvent (THF, $\epsilon=7.6$ ) was considered using the polarisable continuum model (PCM). Single point calculations based on (TD-)DFT geometries were done using the RI-CC2 method. All RI-CC2 computations were performed with the Turbomole v7.0 code. ${ }^{32}$ Since the RI-CC2 method was found to be very sensitive to the 
basis set choice, we used the aug-cc-pVDZ basis set.

The CASPT2 computations were based on configuration state functions obtained with the SA-2-CASSCF $(10,10)$ method. ${ }^{33}$ The active space consisted of eight $\pi$ orbitals with significant occupations and a pair of bonding-antibonding $\sigma(\mathrm{C}-\mathrm{S})$ orbitals (Figure S2, Supporting Information). The CASPT2 calculations were done without an IPEA shift and an imaginary shift of $0.1 \mathrm{au}$, which can solve the problem of possible intruder states. ${ }^{34}$ The excitation energies obtained with CASSCF and CASPT2 methods do not change significantly with an improvement of basis set from 6-31G(d) to aug-cc-pVDZ. Thus, we use the 6-31G(d) basis set for the CASSCF and MS-CASPT2 calculations. The $\mathrm{S}_{1}-\mathrm{S}_{0}$ minimum energy conical intersections (MECIs) were optimised with the SA-2-CASSCF $(10,10) / 6-31 G(d)$ level of theory, using the branching plane update method. ${ }^{35}$ The $T_{1}-S_{0}$ minimum energy crossing points (MECP) were obtained minimising the energy of the $T_{1}$ state with the condition of a zero $\mathrm{T}_{1} / \mathrm{S}_{0}$ gap as implemented in the Molcas code.

We examined the effect of the crystal environment on the excited state mechanisms. First, the experimental crystal structure of TPT, retrieved from the Cambridge Crystallographic Database (the CCDC code is 1494294), ${ }^{12}$ was refined with periodic boundary conditions DFT calculations as implemented in the Quantum Espresso code. ${ }^{36}$ The PBE-D2 functional with dispersion correction was used with a plane-wave cutoff of 30 Ry and a Monkhorst-Pack k-point grid of $(1 \mathrm{x} 2 \mathrm{x} 1)$. These optimisations were performed by relaxing the structure inside unit cell, fixing the cell dimensions to their experimental values.

To analyse intermolecular interactions and calculate exciton couplings, dimers featuring close intermolecular contacts were isolated from the optimised crystal. Exciton couplings $(J)$ at the TD- $\omega$ B97X-D/6-31G(d) level of theory were computed applying a diabatisation method based on the transition dipole moments calculated as implemented in the fromage code. ${ }^{37-39}$ This method takes into account short-range (exchange, orbital overlap, chargetransfer) and long-range Coulomb interactions.

The effect of crystal environment on the excited states was considered using QM/MM 
hybrid methods. A cluster composed of 42 TPT molecules (2058 atoms) was extracted from the optimised supercell. The central molecule in the cluster was treated within the QM framework, whereas the surrounding molecules were treated with MM. The QM region was relaxed, whilst the MM region was kept fixed at its optimised lattice positions. FC, $\mathrm{S}_{1}$, and $\mathrm{T}_{1}$ geometries were optimised applying the ONIOM(QM:MM) method ${ }^{40,41}$ implemented in the Gaussian 16 software. ${ }^{42}$ The QM region was treated at the $\omega$ B97X-D/6-31G(d) and B3LYP/6-31G(d) levels of theory under the (TD-)DFT framework. The MM region was simulated with the Amber force field ${ }^{43}$ and the ESP charges obtained at HF/3-21G* level of theory. The $\mathrm{S}_{1}, \mathrm{~T}_{1}$ minima, $\mathrm{S}_{1}-\mathrm{S}_{0}$ MECI and the $\mathrm{T}_{1}-\mathrm{S}_{0}$ MECP in the solid state were also optimised at the (SA-2-)CASSCF $(10,10) / 6-31 G(d)$ level of theory under the QM/MM additive scheme using the interface between the Molcas and Tinker (version 6.3.3) codes. ${ }^{44}$ The RI-CC2 calculations in the solid state were performed only applying electrostatic embedding.

For a more detailed decay examination between $1^{1} \pi \pi^{*}$ and $1^{1} \pi \sigma^{*}$ minima, we computed the $\mathrm{S}_{0}-\mathrm{S}_{2}$ and $\mathrm{T}_{1}-\mathrm{T}_{3}$ energies along the pathway connecting these geometries. Initially, a set of points was created applying linear interpolation in internal coordinates (LIIC) with the energies calculated at CASPT2/SA-3-CASSCF $(10,10) / 6-31 G(d)$ level of theory. The $\mathrm{S}_{1}$ energy profile along the pathway featured a barrier corresponding to the $\mathrm{S}_{1}\left(\pi \pi^{*}\right) / \mathrm{S}_{1}\left(\pi \sigma^{*}\right)$ crossing. The barriers were decreased by constrained SA-2-CASSCF optimisations of $\mathrm{S}_{1}$ state at several geometries in the vicinity of the crossing.

The spin-orbit coupling matrix elements (SOCME) between the first three singlet and triplet states $\left(\mathrm{S}_{0}-\mathrm{S}_{2}\right.$ and $\left.\mathrm{T}_{1}-\mathrm{T}_{3}\right)$ were computed at the optimised critical points in vacuum and crystal as implemented in the Molcas code. ${ }^{45,46}$ The SOCMEs were obtained from the calculated components corresponding to transitions between a singlet and three spin components defined with quantum numbers $m_{l} \in\{-1,0,1\}$ as $\left|\left\langle S_{x}\left|H_{S O}\right| T_{y}\right\rangle\right|=$ $\sqrt{\sum_{m_{l}=-1,0,1}\left\langle S_{x}\left|H_{S O}\right| T_{m_{l, y}}\right\rangle^{2}}$. To investigate the effect of vibrations on the excited state processes, we calculated the Huang-Rhys factors and reorganisation energies in gas phase, solution, and solid state using the DUSHIN code. ${ }^{47}$ The normal modes of the $\mathrm{S}_{1}$ and $\mathrm{S}_{0}$ minima 
were computed at (TD-) $\omega$ B97X-D/6-31G(d) level of theory.

\section{Results and Discussion}

\section{Vertical excitations and radiative mechanisms}

Table 1 shows the vertical excitations and emission energies of TPT computed with singlereference (TD-DFT and RI-CC2) and the multi-reference MS-CASPT2/CASSCF methods. For the CASPT2 and CC2 calculations, we considered the geometries optimised at the (TD)B3LYP/6-31G(d) level of theory.

Table 1: Excitation energies and oscillator strengths (in parenthesis) of the $\mathrm{S}_{1}$ state TPT molecule in vacuum, solution (THF), and crystal environment. ${ }^{a}$ Value obtained with the aug-cc-pVDZ basis set. ${ }^{b}$ Geometries optimised at the CASSCF /6-31G(d) level of theory.

\begin{tabular}{|c|c|c|c|c|}
\hline & \multicolumn{4}{|c|}{ Energy $(\mathrm{eV})$} \\
\hline & \multicolumn{2}{|c|}{ Vacuum/Solution } & \multicolumn{2}{|c|}{ Crystal } \\
\hline & Absorption & Emission & Absorption & Emission \\
\hline RI-CC2/aug-cc-pVDZ & $4.24(0.40)$ & $3.27(0.61)$ & 4.16 & - \\
\hline TD-B3LYP /6-31G(d) & $3.86(0.33)$ & $2.99(0.43)$ & $3.81(0.57)$ & $3.08(0.60)$ \\
\hline TD- $\omega$ B97X-D/6-31G(d) & $4.43(0.40)$ & $3.28(0.52)$ & $4.32(0.63)$ & $3.30(0.64)$ \\
\hline TD- $\omega$ B97X-D /6-31G(d), PCM & $4.39(0.51)$ & $3.04(0.82)$ & - & - \\
\hline MS-2_CASPT2/6-31G(d) & $4.14 ; 3.84^{a}$ & 3.04 & 3.87 & 3.08 \\
\hline MS-2-CASPT2/0-31G(d) & $4.11^{b}$ & $3.34^{b}$ & $3.90^{b}$ & $2.78^{b}$ \\
\hline Experimental $^{12,48}$ & 3.94 & 3.07 & 3.65 & 3.07 \\
\hline
\end{tabular}

The experimental absorption spectrum of TPT in solution of THF features a maximum at $3.94 \mathrm{eV}$. The maximum of absorption of TPT nanosized aggregates, obtained by adding water to a solution of TPT in THF, shifts to the red (dilution with $90 \mathrm{vol} \% \mathrm{H}_{2} \mathrm{O}$ shifts the maximum to $3.65 \mathrm{eV}$ ). The emission maximum in THF appears at $3.07 \mathrm{eV}$, which position is not affected in the solid phase. ${ }^{12}$

In general, all applied methods provide a reasonable description of the absorption and emission maxima (Table 1). Amongst them, the TD-B3LYP excitation energies are the closest to the experimental values, both in solution and in the crystal phases. The vertical 
excitations computed at the (TD-)B3LYP/6-31G(d) geometries in vacuum and crystal are overestimated for $\sim 0.20 \mathrm{eV}$ with respect to the experimental values. The $\mathrm{S}_{1}$ vertical excitations obtained with RI-CC2 in vacuum and TD- $\omega$ B97X-D in solution are overestimated by 0.30 and $0.45 \mathrm{eV}$ with respect to the experimental value. The emission energies are closer to the experimental values. The TD- $\omega$ B97X-D energies in the solution are underestimated by only $0.03 \mathrm{eV}$. Comparing the TD- $\omega$ B97X-D energies computed in vacuum and in solution, one can notice that the solvent significantly stabilises the $S_{1}$ minimum (for $0.24 \mathrm{eV}$ ), but only slightly affects the vertical excitation. The RI-CC2 emission energy in vacuum is overestimated by $0.20 \mathrm{eV}$. Experimental and computational results show that the crystal environment does not have a significant effect on emission energies. However, it stabilises the $S_{1}$ state at the FC geometry for $\sim 0.3 \mathrm{eV}$.

The stabilisation of vertical excitation by crystal environment is well-reproduced; according to the MS-CASPT2 results the $\mathrm{S}_{1}$ state is stabilised by $\sim 0.27 \mathrm{eV}$ in crystal, while the stabilisation based on experimental peak positions amounts to $\sim 0.29 \mathrm{eV}$. The emission energies computed at TD-DFT optimised geometries are in a very good agreement with experimental ones both in vacuum and crystal (Table 1) and reproduce well the minimal effect of the environment. On the other hand, the energies computed for the CASSCF geometries are overestimated in vacuum and underestimated in crystal for $\sim 0.3 \mathrm{eV}$. Given the important effect of dynamic correlation on geometries, for the study of the FC region and $\pi \pi^{*}$ minima of the PES, we considered the (TD)-DFT geometries.

\section{Exciton couplings and intermolecular interactions}

Specific interactions and exciton couplings can modulate radiative and nonradiative mechanisms. In this section, we analyse intermolecular interactions and exciton couplings in the TPT crystal at ambient pressure. We extracted five dimers (D1-D5) with distances be-

tween the monomer centroids smaller than $10 \AA$ from the refined crystal structure. The closest contacts between the molecules are C-H.. $\pi$, C-H..C, and H..H interactions. The $\pi-\pi$ 
interactions between phenyl rings are not very effective because of the in-plane slip of the monomers. $\mathrm{Gu}$ et al. have observed a significant piezoresponsive luminescence in the range of pressures between $\sim 1$ and $5.7 \mathrm{GPa}$. The enhance of fluorescence was attributed to the strengthening of C-H.. $\pi$ and C-H...C intermolecular interactions that hinder the rotation of phenyl rings increasing the radiative response. Further increase of external pressure increases the intermolecular $\pi . . \pi$ interactions decreasing the radiative response. ${ }^{13}$

The dimer with the shortest centroid distance is the slip-stacked D1. The smallest intermolecular distance is a H..H contact at $2.12 \AA$, the same kind of interaction is also found in D2 at $3.83 \AA$. Monomers belonging to D4 and D5 dimers interact via C-H..C between the phenyl rings. For the V-shaped D3, the shortest intermolecular C..H contact has a length of $2.93 \AA$ (Figure 2). When the external pressure is applied, this distance decreases and new CH.. $\pi$ interactions are stabilised. ${ }^{13}$ For instance, the corresponding C..H length drops to 2.42 $\AA$ at 9 GPa. ${ }^{13}$ Apart from that, the C-H.. $\pi$ bond formation is also demonstrated by a blueshift of the aromatic C-H stretching band observed at high pressures. These intermolecular interactions hinder low frequency vibrations, which has an impact on nonradiative decay.

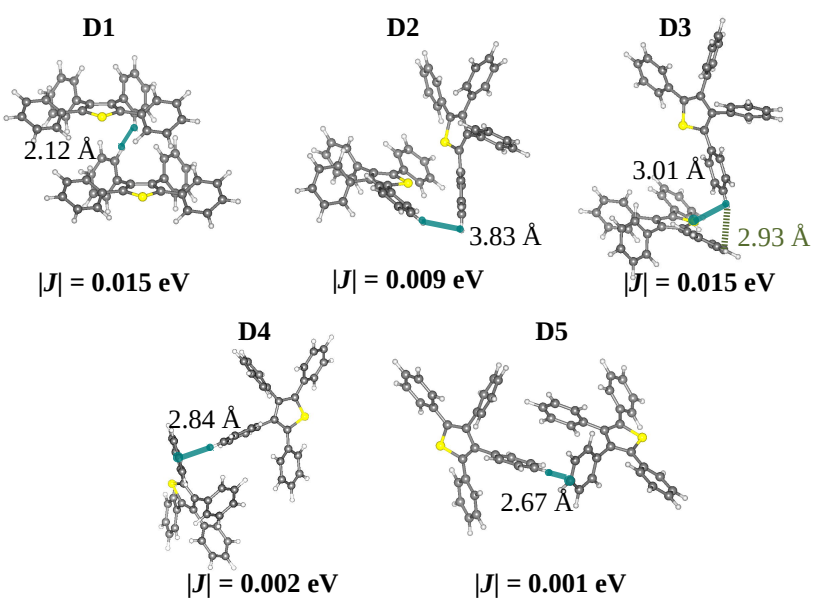

Figure 2: Structures of TPT dimers showing the closest intermolecular contacts $(\AA)$ and absolute values of the exciton couplings (in $\mathrm{eV}$ ).

There is not a significant overlap between electron densities of monomers (SI, Section S3), therefore the exciton couplings are very small (0.001- 0.015 eV, Figure 2) in comparison 
with the reorganisation energies $(0.52 \mathrm{eV}$, see next section). This indicates that the excited state processes are mainly localised on the independent molecular units. The slip-stacked D1 and herringbone D3 dimers feature the largest $J$ values $(0.015 \mathrm{eV})$. The monomer slipping decreases the contact between the densities and consequently the exciton couplings are small. $n-\pi$ interactions between Sulphur and thiophene ring do not affect exciton couplings, because Sulphur does not contribute to the $\mathrm{S}_{1}$ transition.

\section{Restriction of intramolecular vibrations in the solid state}

According to the RIR interpretation of AIE, low energy vibrations are restricted in the solid state. To analyse the behaviour of TPT in different phases, we calculated the reorganisation energies $(\lambda)$ (Figure 3). The $\lambda$ values for the $S_{1}$ to $S_{0}$ transition are $5281 \mathrm{~cm}^{-1}(0.65 \mathrm{eV}), 5820$ $\mathrm{cm}^{-1}(0.72 \mathrm{eV})$, and $4188 \mathrm{~cm}^{-1}(0.52 \mathrm{eV})$ in vacuum, THF solution, and crystal, respectively. The computed $\lambda$ in vacuum is somewhat larger than one obtained by Nie et al. ${ }^{12}\left(4107 \mathrm{~cm}^{-1}\right)$ based on the TD-B3LYP/6-31G(d,p) calculations.
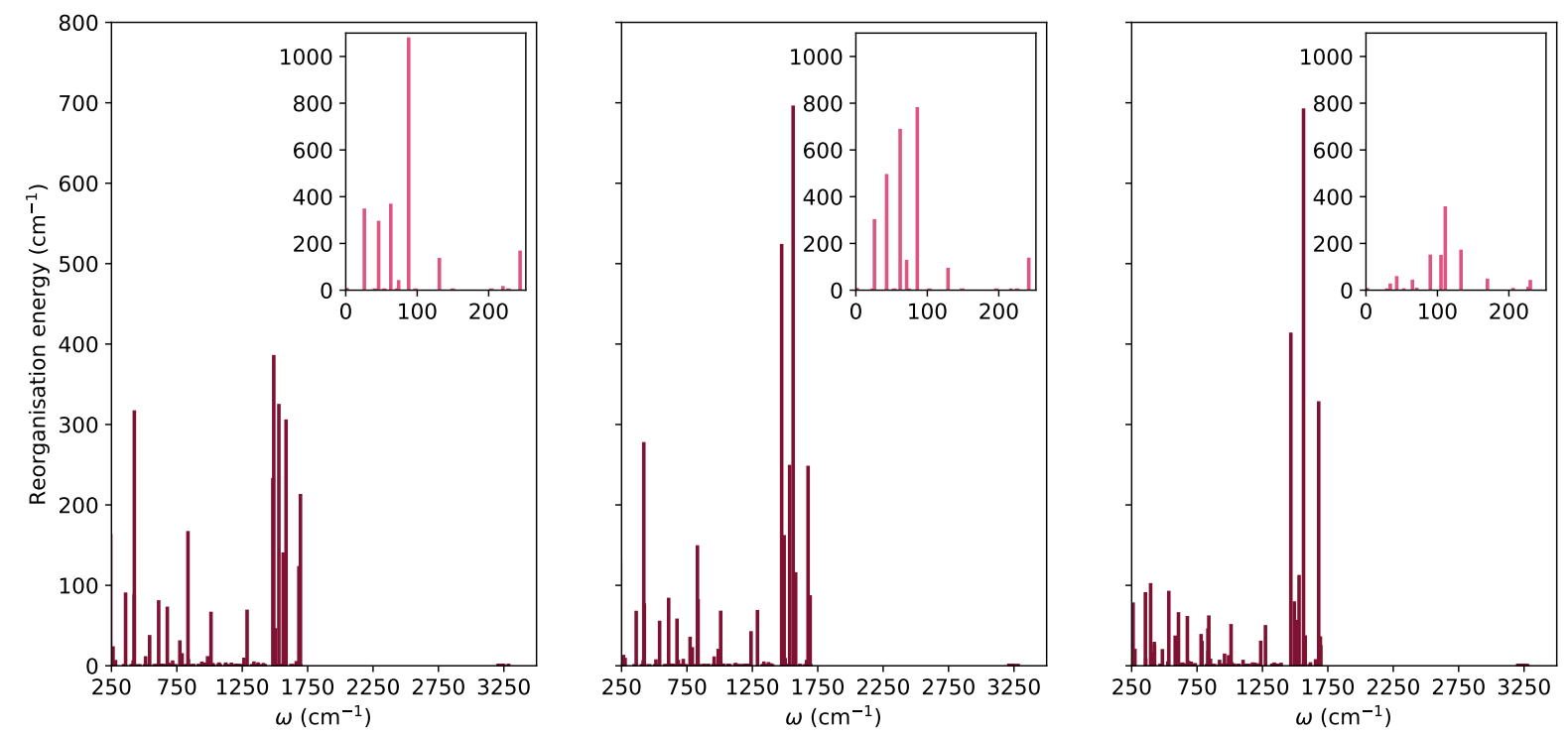

Figure 3: Reorganisation energies $\left(\right.$ in $\mathrm{cm}^{-1}$ ) for the $\mathrm{S}_{1}-\mathrm{S}_{0}$ transition in, from left to right, gas phase, solution, and solid state.

The reorganisation energies decrease going from vacuum (solution) $\left(5281 \mathrm{~cm}^{-1}, 5820\right.$ 
$\left.\mathrm{cm}^{-1}\right)$ to crystal $\left(4188 \mathrm{~cm}^{-1}\right)$ due to the restriction of low-frequency normal modes $(\omega<$ $\left.250 \mathrm{~cm}^{-1}\right)$, such as collective rotations of phenyl groups that are sterically hindered in the crystal. The total reorganisation energy of the low-frequency modes decreases from 2428.4 $\mathrm{cm}^{-1}$ in vacuum and $2597.5 \mathrm{~cm}^{-1}$ in solution to $1210.1 \mathrm{~cm}^{-1}$ in crystal. Additionally, the contribution of higher frequency modes (bending and stretching) to the total reorganisation energies is not significantly altered by environment (their total contributions are 2852 $\mathrm{cm}^{-1}, 3222 \mathrm{~cm}^{-1}$, and $2978 \mathrm{~cm}^{-1}$ in vacuum, solution and crystal). This distribution of $\lambda$ indicates that in the crystal phase, low-frequency torsional modes are less prone to accept excess vibrational energy during relaxation from $\mathrm{S}_{1}$ to $\mathrm{S}_{0}$ compared to vacuum and solution. Accordingly, the relaxation processes involving large amplitude torsions will be hindered in crystal. In contrast, the stretching modes have similar reorganisation energies in all three media, implying that processes involving $\mathrm{C}-\mathrm{S}$ and $\mathrm{C}-\mathrm{C}$ bond elongation will not be significantly perturbed by crystal environment. We show in the next sections, that the main deactivation mechanisms in this crystal are associated with C-S breaking.

\section{Excited State Relaxation Pathways}

To understand the effect of aggregation on the emissive response of $\mathbf{T P T}$, we investigate the radiative pathways for singlet and triplet states in the gas and crystalline phases. The bright state $\left(\mathrm{S}_{1}\right)$ and $\mathrm{T}_{1}$ are $\pi \pi^{*}$ states and have very weak SOC. ${ }^{12}$ However, the distortion of the geometry along the de-excitation pathways allows the mixing of states with different diabatic character resulting in significant SOCs.

\section{Vacuum}

Figure 4 shows the CASPT2/6-31G(d) energies at the critical points relevant for the nonradiative mechanisms in vacuum. The structures with small multiconfigurational character, such as the FC, $\mathrm{S}_{1}$ and $\mathrm{T}_{1}$ minima, were obtained from (TD)-DFT calculations, while those with a pronounced multireference nature including the crossing geometries $\left(\mathrm{S}_{0} / \mathrm{S}_{1}\right.$ and $\left.\mathrm{S}_{0} / \mathrm{T}_{1}\right)$ 
and the $\pi \sigma^{*}$ minima were optimised at (SA-2-)CASSCF $(10,10) / 6-31 \mathrm{G}(\mathrm{d})$ level of theory. A diagram compiling the energies of all (SA-2-)CASSCF $(10,10) / 6-31 \mathrm{G}(\mathrm{d})$ geometries can be found in the SI.

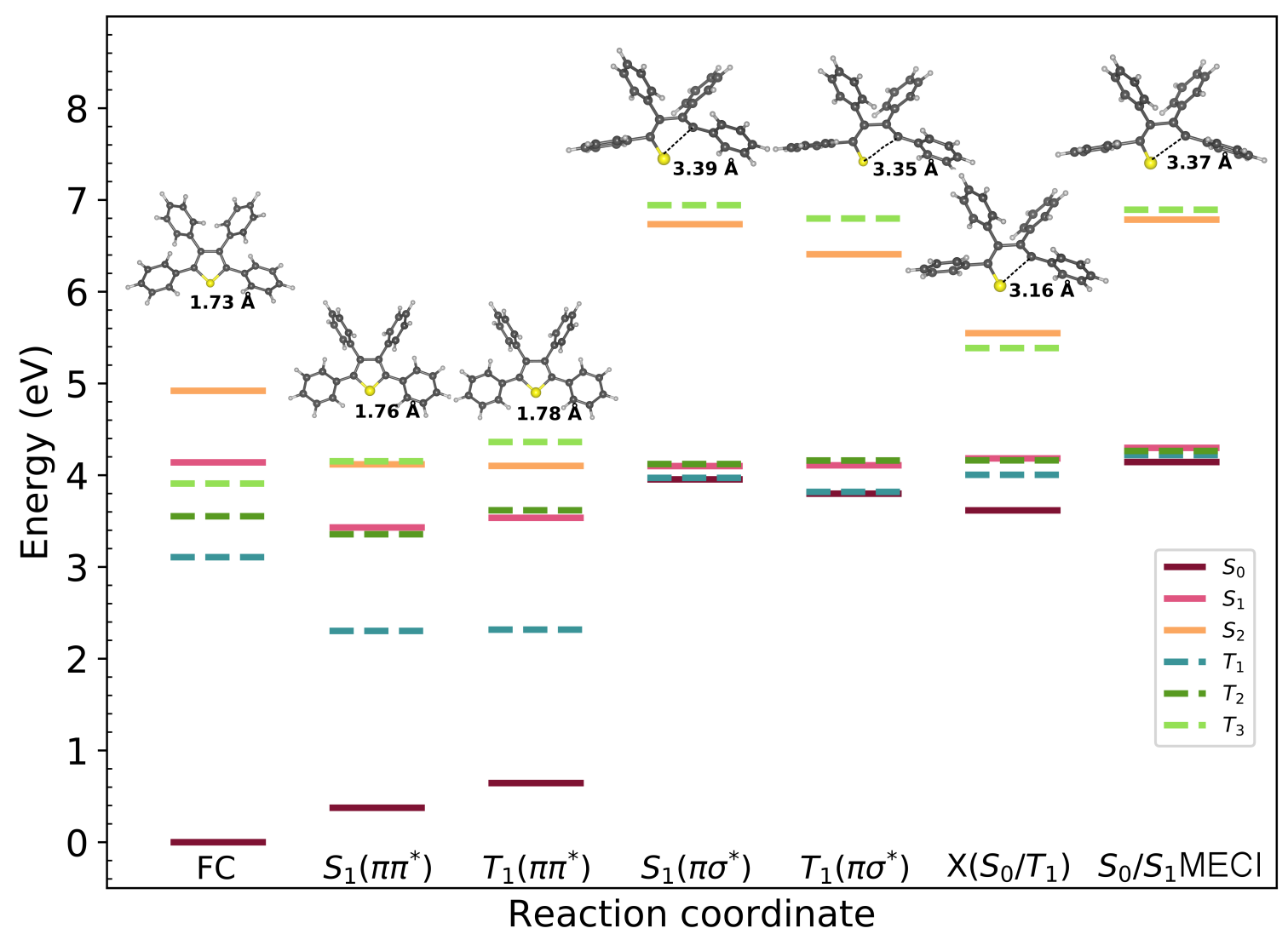

Figure 4: CASPT2/SA-3-CASSCF $(10,10) / 6-31 G(d)$ energies at the critical points in vacuum.

In contrast with analogous propeller systems (DMTPS, TPC and others) ${ }^{7,10}$ deactivated through ring puckering conical intersections, the main nonradiative pathways in TPT involve C-S bond breaking. The mechanism is similar to the observed in thiophene and its derivatives in vacuum, which involves both $\mathrm{S}_{1}$ minima: ${ }^{1} \pi \pi^{*}$ and ${ }^{1} \pi \sigma^{*} .{ }^{49-54}$ In the first step, the molecule relaxes to the ${ }^{1} \pi \pi^{*}$ minimum ( $0.72 \mathrm{eV}$ below $S_{1}$ at the FC geometry). The C-S stretching modes, among the others, gain kinetic energy enabling the C-S elongation and ring-opening (Figure 4$). \mathrm{S}_{1}\left(\pi \pi^{*}\right)$ and $\mathrm{T}_{2}\left(\pi \pi^{*}\right)$ states are nearly degenerate at the $\mathrm{S}_{1}$ minimum (Figure 4). Both the FC and $\pi \pi^{*}$ minima are planar. Our calculations show that C-S stretching 
leads to the transition from ${ }^{1} \pi \pi^{*}$ to the ${ }^{1} \pi \sigma^{*}$ state.

In the region of the potential energy surface around the ${ }^{1} \pi \sigma^{*}$ state minimum, ${ }^{1} \pi \sigma^{*}$ state is highly degenerate, and the $\mathrm{T}_{1}\left(\pi \sigma^{*}\right)$ minimum, $\mathrm{X}\left(\mathrm{S}_{0} / \mathrm{T}_{1}\right)$ and $\mathrm{S}_{0} / \mathrm{S}_{1}$ MECI crossing structures are in close proximity (Figure 4). The crossing structures of TPT share some similarities with those found for thiophene. The C-S distance in the $\mathrm{S}_{0} / \mathrm{S}_{1}$ MECI in thiophene is 3.40 $\AA,{ }^{50}$ a value close to the one in TPT $(3.37 \AA)$. However, in contrast to the planarity of the thiophene structures, the $\mathrm{S}_{0} / \mathrm{S}_{1}$ MECI of TPT deviates significantly from the plane $\left(\angle C C C C=67.3^{\circ}\right.$, Figure 5). ${ }^{49}$ The $\mathrm{S}_{0} / \mathrm{S}_{1}$ MECI has a biradical structure with unpaired electrons at terminal $\mathrm{S}$ and $\mathrm{C}$ atoms. In the case of $\mathrm{X}\left(\mathrm{S}_{0} / \mathrm{T}_{1}\right)$ crossing, it features a significant contribution from the $\mathrm{C} 2-\mathrm{C} 3 \pi$ orbital, resulting in a smaller deviation from planarity $\left(\angle S C C C=20.6^{\circ}\right)$ (Figure 5). The $\mathrm{S}_{0} / \mathrm{S}_{1} \mathrm{MECI}$ lies $0.16 \mathrm{eV}$ above the $1^{1} \pi \sigma^{*}$ minimum and $0.07 \mathrm{eV}$ above the initial excitation level, and internal conversion can happen for a wide range of geometries with C-S distances, from $3.10 \AA$ to $3.37 \AA$ ) (Figure 4). Thermal fluctuations could trigger the relaxation to the ground state before the $\mathrm{S}_{0} / \mathrm{S}_{1}$ MECI geometry is reached.

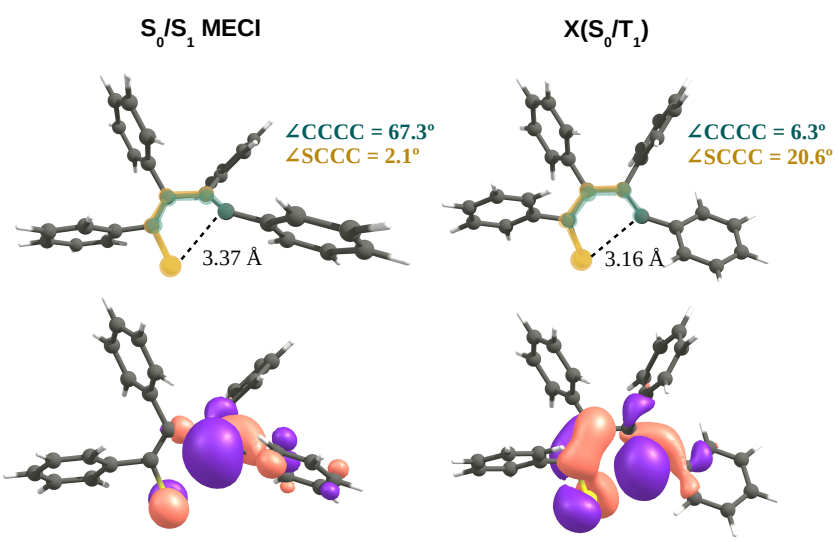

Figure 5: Geometries of $\mathrm{S}_{0} / \mathrm{S}_{1}$ and $\mathrm{S}_{0} / \mathrm{T}_{1}$ minimum energy crossing points in vacuum optimised at the (SA-2-)CASSCF $(10,10) / 6-31 \mathrm{G}(\mathrm{d})$ level. CASPT2/CASSCF $(10,10) / 6-31 \mathrm{G}(\mathrm{d})$ density differences of $\mathrm{S}_{1}$ and $\mathrm{T}_{1}$ states are given below.

To better understand the relaxation mechanism, we analyse the pathways between the FC geometry going through the $\mathrm{S}_{1}\left(\pi \pi^{*}\right)$ to the $\mathrm{S}_{1}\left(\pi \sigma^{*}\right)$ minimum. Figure 6 shows the linear interpolated profiles between the ${ }^{1} \pi \pi^{*}$ and ${ }^{1} \pi \sigma^{*}$ minima under the adiabatic and diabatic 
representations. We also analysed the $\mathrm{SOC}$ values between singlets and triplets (Figure 7). Based on the transformation of $\mathrm{S}_{1}, \mathrm{~T}_{1}$ and $\mathrm{T}_{2}$ states, the whole region can be divided into four domains (regions I-IV) (Figure 7). Given the similarity in energies and significant values of the spin-orbit couplings, both internal conversion and intersystem crossing are relevant for the quenching of emission in non-aggregated phases.
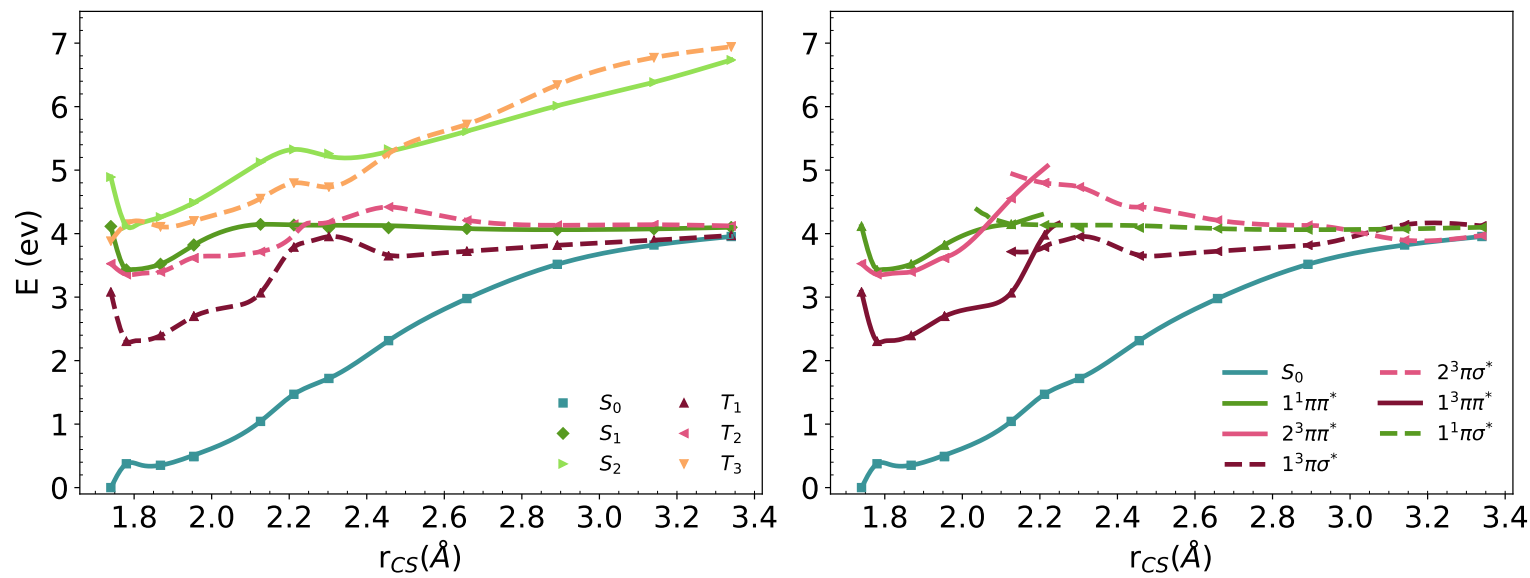

Figure 6: CASPT2/SA-3-CASSCF $(10,10) / 6-31 \mathrm{G}(\mathrm{d})$ energies along the LIIC pathway connecting $\mathrm{S}_{0}, \mathrm{~S}_{1}\left(\pi \pi^{*}\right)$ and $\mathrm{S}_{1}\left(\pi \sigma^{*}\right)$ minima in vacuum. The adiabatic (left) and diabatic representations (right) are shown.

In the first region $\left(1.8 \AA \lesssim r_{C S} \lesssim 2.1 \AA\right)$, which is in the vicinity of the ${ }^{1} \pi \pi^{*}$ minimum, the $\mathrm{S}_{1}, \mathrm{~T}_{1}$, and $\mathrm{T}_{2}$ are $\pi \pi^{*}$ states, resulting in very low spin-orbit couplings. The transition from the $\mathrm{S}_{1}\left(\pi \pi^{*}\right)$ to the $\mathrm{S}_{1}\left(\pi \sigma^{*}\right)$ minimum is facilitated by a crossing between them at $\sim 2.1 \AA$, which is accessible provided the initial excitation energy (barrier $0.6 \mathrm{eV}$ ). Further C-S stretching $\left(2.1 \AA \lesssim r_{C S} \lesssim 2.6 \AA\right.$, region II) increases the spin-orbit couplings. In region II, thiophene ring maintains its planarity and the $S_{1}$ and $T_{1}$ states have mainly $\pi \sigma^{*}$ character with a minor $\pi \pi^{*}$ contribution that vanishes with stretching. The $\mathrm{T}_{2}$ state preserves $\pi \pi^{*}$ character, and gradually gains a small $\pi \sigma^{*}$ contribution. The $\mathrm{S}_{1} / \mathrm{T}_{2}$ couplings $\left(\left\langle\pi \sigma^{*}\left|\hat{H}_{S O}\right| \pi \pi^{*}\right\rangle\right)$ slightly decrease as $\mathrm{T}_{2}$ gains larger $\pi \sigma^{*}$ contribution. The $\mathrm{S}_{1} / \mathrm{T}_{1}$ couplings are smaller and decrease with stretching as both states converge to pure $\pi \sigma^{*}$ character.

For a range of C-S distances $(>2.4 \AA)$, the $1^{1} \pi \sigma^{*}, 1^{3} \pi \sigma^{*}, 2^{3} \pi \sigma^{*}$ and the ground state have similar energies and significant SOCs opening the possibility of relaxation through the 
intersystem crossing channels. The $1^{1} \pi \sigma^{*}, 1^{3} \pi \sigma^{*}$ and $2^{3} \pi \sigma^{*}$ states cross at a C-S distance of around $3.1 \AA$, the spin-orbit couplings are larger than $70 \mathrm{~cm}^{-1}$ for both pairs of states (Figure 7). At the $1^{1} \pi \sigma^{*}$ minimum, the spin-orbit couplings with $2^{3} \pi \sigma^{*}$ and $1^{3} \pi \sigma^{*}$ states are $157.7 \mathrm{~cm}^{-1}$ and $61.7 \mathrm{~cm}^{-1}$ respectively (section $\mathbf{S 6}$ in SI). Both states can decay to the ground state through the accessible $1^{3} \pi \sigma^{*} / \mathrm{S}_{0}$ crossing, located $0.34 \mathrm{eV}$ below the energy of the $\mathrm{S}_{1}$ excitation at the FC point (Figure 4). A small spin-orbit coupling of $5.4 \mathrm{~cm}^{-1}$ is associated with this transition (section $\mathbf{S 6}$ in SI).

The C-S stretching between $2.6 \AA$ and $2.9 \AA$ (region III) is followed by a significant outof-plane torsion of thiophene (Figure 7). The three states can be described as $\pi \sigma^{*}$ excitations with significant biradical character. The terminal $\mathrm{C}$ has a larger density than the $\mathrm{S}$ atom. The $\mathrm{S}_{1}, \mathrm{~T}_{1}$, and $\mathrm{T}_{2}$ densities have similar spatial orientations around $\mathrm{C}$ atom, however the p-orbital of the $\mathrm{S}$ atom in the $\mathrm{S}_{1}$ state is roughly perpendicular to the ones in $\mathrm{T}_{1}$ and $\mathrm{T}_{2}$ states, which determines the important SOCs between both $\mathrm{S}_{1} / \mathrm{T}_{1}$ and $\mathrm{S}_{1} / \mathrm{T}_{2}$ pairs.

The C-S stretching in the region of $2.9 \AA \lesssim r_{C S} \lesssim 3.35 \AA$ (region IV) preserves the biradical characters of $S_{1}$ and $T_{1}$ with a larger density on the $\mathrm{S}$ atom. Both states have similar densities and the SOC values between them become quite small. The $\mathrm{T}_{2}$ gains some $\pi \pi^{*}$ contribution which increases the $\mathrm{SOC}$ with the $\mathrm{S}_{1}$ state $\left(\pi \sigma^{*}\right.$ state). When the geometry approaches to the ${ }^{1} \pi \sigma^{*}$ minimum, the contribution of double excitations becomes more important. Once the molecule approaches the multiconfigurational region, internal conversion or intersystem crossing are possible at different intermolecular distances. Internal conversion from $S_{1}$ to $S_{0}$ is expected to be faster than intersystem crossing from $T_{1}$ to $S_{0}$. However, intersystem crossing from $S_{1}$ to $T_{1}$ and $T_{2}$ could be very effective because of the large values of SOCs. If molecule is on the $T_{1}$ surface, crossing to $S_{0}$ can happen at several geometries along the crossing seam and the $\mathrm{X}\left(\mathrm{T}_{1} / \mathrm{S}_{0}\right)$ is classically accessible (Figure 4$)$. All these mechanisms will lead to emission quenching.

Schnappinger et al. have explored the nonadiabatic dynamics of thiophene considering both singlet and triplet states. ${ }^{53}$ They found that along the ring-opening coordinate, the 

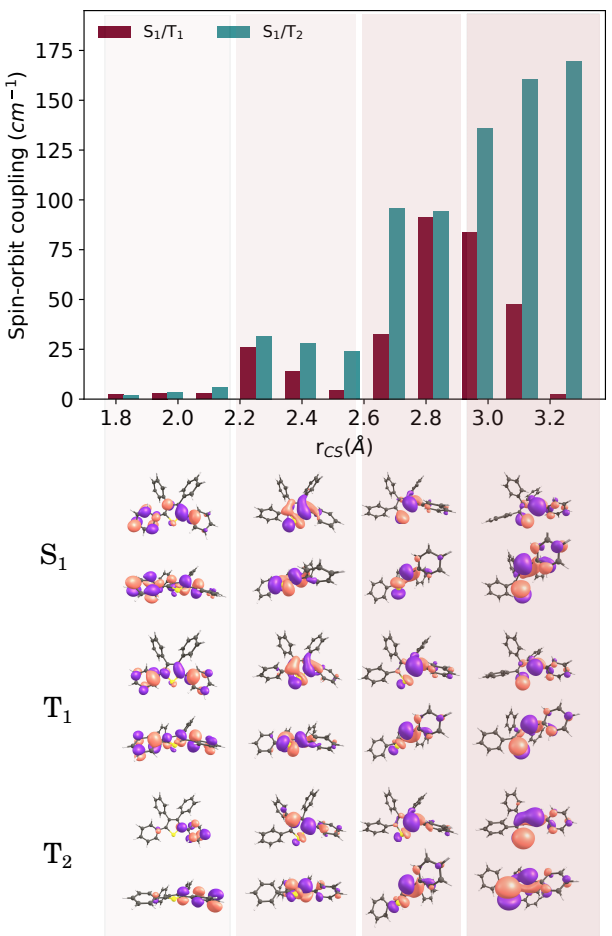

Figure 7: Spin-orbit couplings (in $\mathrm{cm}^{-1}$ ) between $\mathrm{S}_{1} / \mathrm{T}_{1}$ and $\mathrm{S}_{1} / \mathrm{T}_{2}$ adiabatic states along the LIIC pathway between $\mathrm{S}_{1}\left(\pi \pi^{*}\right)$ and $\mathrm{S}_{1}\left(\pi \sigma^{*}\right)$ minima computed at the CASPT2 level in vacuum. $\mathrm{S}_{1}, \mathrm{~T}_{1}$, and $\mathrm{T}_{2}$ CASPT2 density differences are given in two orthogonal perspectives. 
role of triplets becomes very important, competing with internal conversion. In contrast with the mechanism for thiophene, where the ${ }^{1} \pi \sigma^{*}$ minimum is the global minimum and the system can get trapped in open-ring structures, in TPT trapping in ${ }^{1} \pi \sigma^{*}$ minimum is unlikely, because the ${ }^{1} \pi \sigma^{*}$ well is very shallow and higher in energy than the ${ }^{1} \pi \pi^{*}$ minimum. Since the molecule can also go back from the multiconfigurational to closed-ring region, it might be still be possible to obtain weak fluorescence and phosphorescence from ${ }^{1} \pi \pi^{*}$ and ${ }^{3} \pi \pi^{*}$ minima respectively. In a recent paper the dynamics of a related molecule (2-methyl-5-

phenylthiophene) was also explored using stimulated Raman scattering with transient singlet state absorption and TDDFT calculations. ${ }^{55}$ The authors observed the time constants that were correlated with the activation of C-S stretching followed by intersystem crossing to the triplet state. The ISC was attributed to a $\mathrm{S}_{1} / \mathrm{T}_{2}$ transition, followed by a rapid internal conversion to the $\mathrm{T}_{1}$ state.

\section{Crystal}

In this Section, we examine the effect of crystal environment on the excited state mechanism of TPT. Figure 8 shows the CASPT2 energies at the stationary and crossing geometries. We have also built the pathway connecting the $\mathrm{FC}$ point, ${ }^{1} \pi \pi^{*}$, and ${ }^{1} \pi \sigma^{*}$ minima (Figure 9). Figure 10 shows the values of SOCs along the C-S stretching coordinate (also see Table 4 in SI). Similarly to vacuum, the ${ }^{1} \pi \sigma^{*}$ minimum is less stable than the ${ }^{1} \pi \pi^{*}$ minimum and classically accessible.

The $\mathrm{S}_{1}, \mathrm{~T}_{1}$ and $\mathrm{T}_{2}$ states are nearly degenerate at the ${ }^{1} \pi \sigma^{*}$ minimum (Figure 8) with SOCs of $\sim 25 \mathrm{~cm}^{-1}$ (Figure 10). The ${ }^{1} \pi \sigma^{*}$ state is dark (oscillator strength of 0.05 ) so the fluorescence from this state should be negligible. In contrast with vacuum, where the $\mathrm{S}_{1}-\mathrm{S}_{0}$ gap at the ${ }^{1} \pi \sigma^{*}$ minimum is small $(0.15 \mathrm{eV})$, it is $\sim 2 \mathrm{eV}$ in the solid state. The crystal restricts relaxation and the the $\mathrm{S}_{0} / \mathrm{S}_{1}$ MECI is less distorted in the solid state with an energy of $0.7 \mathrm{eV}$ above the initial $\mathrm{S}_{1}$ excitation. Consequently, the $\mathrm{S}_{0} / \mathrm{S}_{1} \mathrm{MECI}$ is classically inaccessible provided excitation to the $\mathrm{S}_{1}$ state and internal conversion to the ground state 


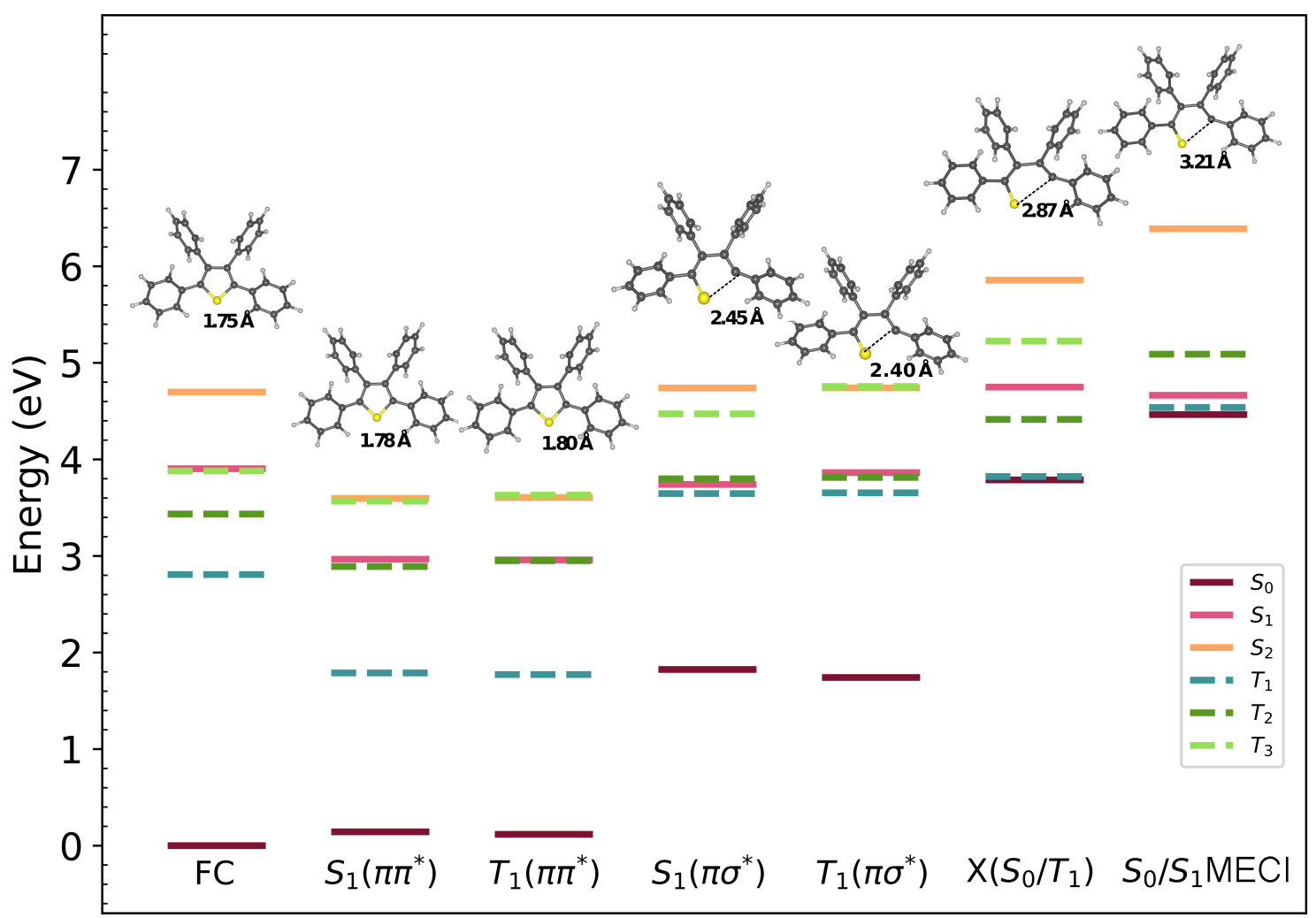

Reaction coordinate

Figure 8: CASPT2/SA-3-CASSCF (10,10)/6-31G(d) energies of $\mathrm{S}_{0}-\mathrm{S}_{2}$ and $\mathrm{T}_{1}-\mathrm{T}_{3}$ states at the critical points optimised at the TD-B3LYP/6-31G(d) level $\left(\mathrm{S}_{0}, \mathrm{~S}_{1}\right.$, and $\mathrm{T}_{1}$ geometries) and at the (SA-2-)CASSCF $(10,10) / 6-31 \mathrm{G}(\mathrm{d})$ level $\left(\pi \sigma^{*}\right.$ states and crossing points) in crystal.
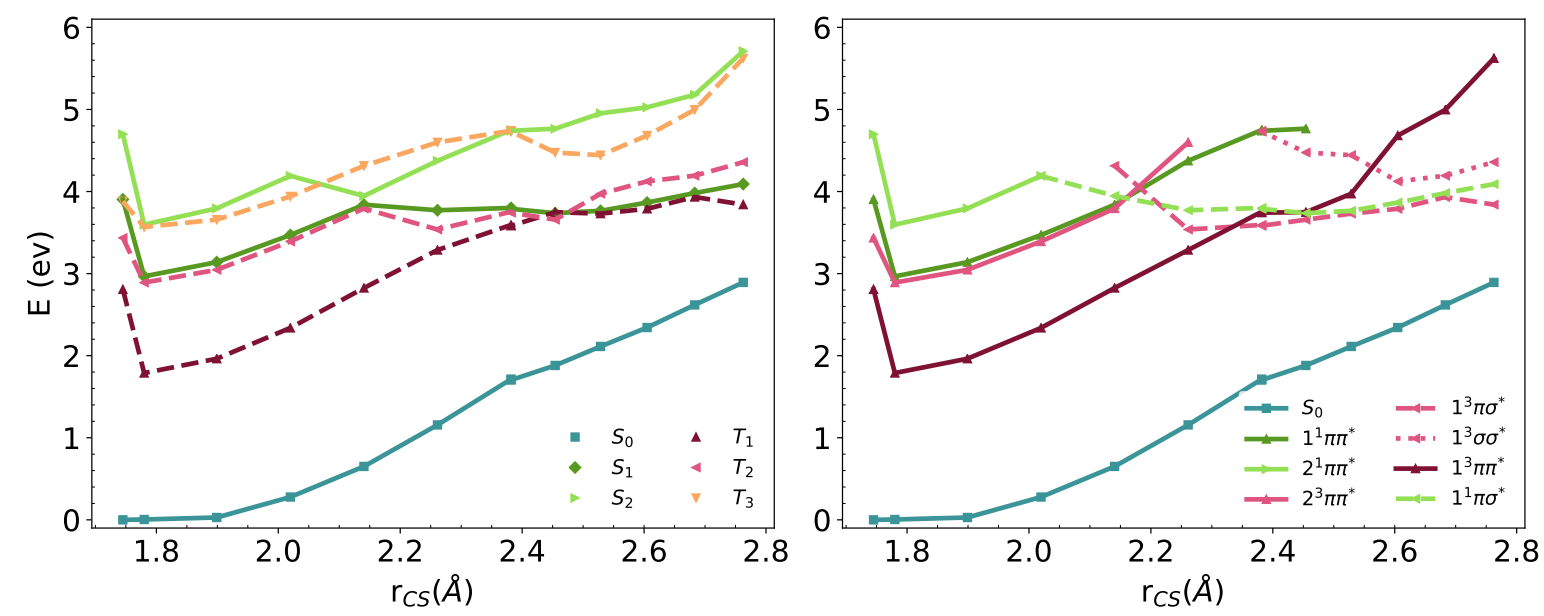

Figure 9: CASPT2/SA-3-CASSCF $(10,10) / 6-31 \mathrm{G}(\mathrm{d})$ energies of $\mathrm{S}_{0}-\mathrm{S}_{2}$ and $\mathrm{T}_{1}-\mathrm{T}_{3}$ states along the LIIC pathway connecting $\mathrm{S}_{0}, \mathrm{~S}_{1}\left(\pi \pi^{*}\right)$, and $\mathrm{S}_{1}\left(\pi \sigma^{*}\right)$ minima in crystal in adiabatic (left) and diabatic representation (right). 
is hindered in the crystal. This is in line with the RACI model for AIE in the solid state. ${ }^{5}$ However, reaction channels involving intersystem crossing could be still available in the solid state.

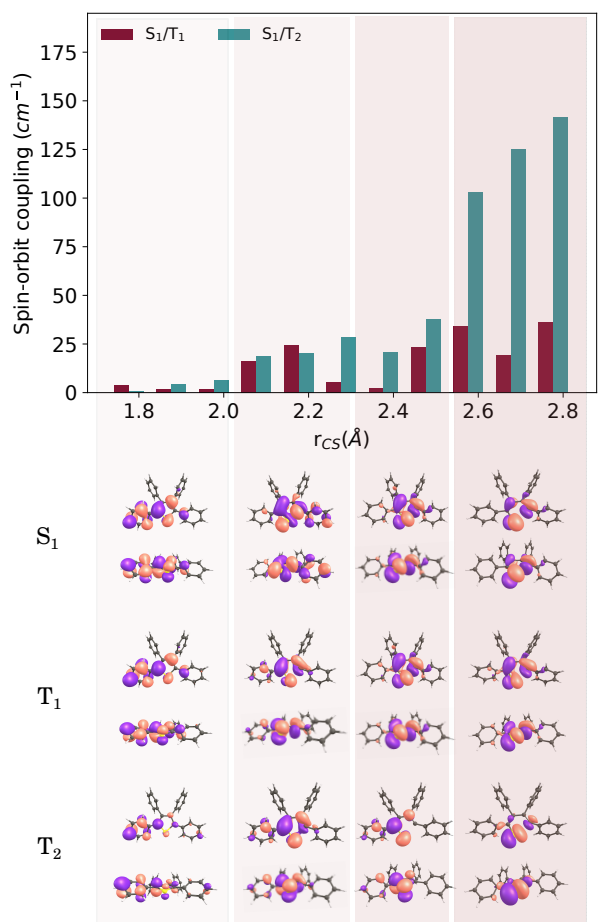

Figure 10: Spin-orbit couplings (in $\mathrm{cm}^{-1}$ ) between $\mathrm{S}_{1} / \mathrm{T}_{1}$ and $\mathrm{S}_{1} / \mathrm{T}_{2}$ adiabatic states along the LIIC pathway between $\mathrm{S}_{1}\left(\pi \pi^{*}\right)$ and $\mathrm{S}_{1}\left(\pi \sigma^{*}\right)$ minima computed at the CASPT2 level in crystal. $\mathrm{S}_{1}, \mathrm{~T}_{1}$, and $\mathrm{T}_{2}$ CASPT2 density differences are given in two orthogonal perspectives.

Similar to vacuum, the relaxation pathway can be split into four regions based on nature of the states (Figure 10). Because of the geometrical restrictions imposed in the solid state, SOCs are sligthly smaller than in vacuum. The energy profiles in the region I $\left(r_{C S} \lesssim 2.15\right.$ $\AA$ ) are very similar to the ones in vacuum (Figure 9). The $S_{1}, T_{1}$, and $T_{2}$ states have $\pi \pi^{*}$ character and exhibit weak spin-orbit coupling. The ${ }^{1} \pi \pi^{*} /{ }^{1} \pi \sigma^{*}$ intersection occurs at $\sim 2.15$ $\AA$ and lies $0.1 \mathrm{eV}$ below the ${ }^{1} \pi \pi^{*}$ state in the $\mathrm{FC}$ region. Comparable to the vacuum, in the region II $\left(2.15 \AA \lesssim r_{C S} \lesssim 2.3 \AA\right)$, the $\mathrm{S}_{1}$ and $\mathrm{T}_{1}$ are $\pi \sigma^{*}$ states and are perpendicular to $\mathrm{T}_{2}\left(\pi \pi^{*}\right)$ state. The significant $\mathrm{S}_{1} / \mathrm{T}_{2}$ spin-orbit couplings and small energy gaps make ISC possible in this region. However, the ISC competes with relaxation on ${ }^{1} \pi \sigma^{*}$ surface that 
stabilises with stretching.

The ${ }^{1} \pi \sigma^{*}$ state has a broad minimum between $2.3 \AA \lesssim r_{C S} \lesssim 2.55 \AA$ in crystal. The $\mathrm{T}_{2}$ preserves the $\pi \pi^{*}$ nature, and $\mathrm{S}_{1}$ and $\mathrm{T}_{1}$ states the $\pi \sigma^{*}$ characters. The $\mathrm{S}_{1} / \mathrm{T}_{2}$ and $\mathrm{S}_{1} / \mathrm{T}_{1}$ spin-orbit couplings are $39 \mathrm{~cm}^{-1}$ and $23 \mathrm{~cm}^{-1}$ at $2.55 \AA$. The $\mathrm{S}_{1} / \mathrm{T}_{2}$ ISC can be followed by internal conversion $\left(\mathrm{T}_{2} / \mathrm{T}_{1}\right)$. Furthermore, intersystem crossing can bring the molecule to the ground state, via the $\mathrm{X}\left(\mathrm{S}_{0} / \mathrm{T}_{1}\right)$ crossing with a C-S distance of $2.87 \AA$, lying $0.2 \mathrm{eV}$ below the $\mathrm{S}_{1}$ state in the $\mathrm{FC}$ region (Figure 8 ). This step is allowed by a moderate $\mathrm{T}_{1} / \mathrm{S}_{0}$ spin-orbit coupling $\left(11.9 \mathrm{~cm}^{-1}\right.$, section $\mathbf{S 6}$ in SI)

In the last region $\left(2.55 \AA \lesssim r_{C S} \lesssim 2.8 \AA\right)$ the energies of three states slightly increase, remaining almost degenerate, but the excess energy is sufficient to explore this domain. The $\mathrm{S}_{1}$ and $\mathrm{T}_{1}$ states retain $\pi \sigma^{*}$ character with small $\pi \pi^{*}$ contribution, while $\mathrm{T}_{2}$ state has $\sigma \sigma^{*}$ character, due to $\pi \pi^{*} / \sigma \sigma^{*}$ crossing at $\sim 2.55 \AA$. Orthogonal transitions induce very large $\mathrm{S}_{1} / \mathrm{T}_{2}$ spin-orbit couplings $\left(110 \mathrm{~cm}^{-1}-160 \mathrm{~cm}^{-1}\right.$, Figure 10) and an efficient $\mathrm{S}_{1} / \mathrm{T}_{2}$ ISC is expected. After the ISC, the $\sigma \sigma^{*}$ state stabilises by a slight C-S contraction, followed by back-transition to the ${ }^{3} \pi \pi^{*}$ state at $\sim 2.55 \AA$. From here the decay can be followed by relaxation to $2.3 \AA-2.5 \AA$ region, subsequent internal conversion to ${ }^{3} \pi \sigma^{*}$ state, and ${ }^{3} \pi \sigma^{*} / \mathrm{S}_{0}$ transition, as explained before.

The difference between the photochemistry in the gas phase and the solid state can be understood by analysing the $\mathrm{S}_{0} / \mathrm{S}_{1} \mathrm{MECI}$ and the $\mathrm{X}\left(\mathrm{S}_{0} / \mathrm{T}_{1}\right)$ crossing structures (Figure 11). In the solid state, quenching due to internal conversion to $S_{0}$ is blocked, while decay through intersystem crossing is still possible. Since the crystal environment forces more planar geometries, the $\mathrm{S}_{0} / \mathrm{S}_{1}$ MECI experiences a larger electrostatic repulsion than in $\mathrm{X}\left(\mathrm{S}_{0} / \mathrm{T}_{1}\right)$. The density plot shows a direct interaction between the two antibonding densities which destabilises the $\mathrm{S}_{0} / \mathrm{S}_{1}$ MECI. In the case of $\mathrm{X}\left(\mathrm{S}_{0} / \mathrm{T}_{1}\right)$, the $\pi$ density over C2-C3 atoms enhances compared to the vacuum, and $\mathrm{p}$ densities on the terminal $\mathrm{C}$ and $\mathrm{S}$ atoms are perpendicular to the molecular plane. Our calculations show that while nonradiative decay will be more efficient in the gas phase since both internal conversion and ISC are possible, only the last 
mechanism can be activated in the solid state. This is a plausible explanation for the weak AIE observed for TPT in comparison with other propeller-shaped molecules.

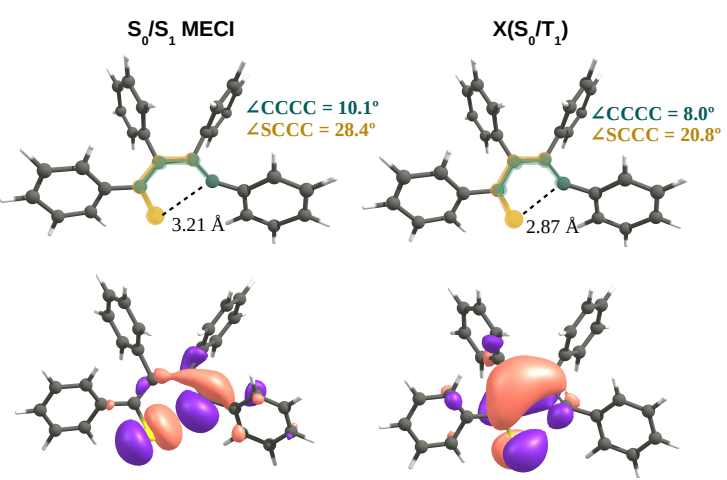

Figure 11: Geometries of $\mathrm{S}_{0} / \mathrm{S}_{1}$ and $\mathrm{S}_{0} / \mathrm{T}_{1}$ minimum energy crossing points in crystal optimised at the (SA-2-)CASSCF $(10,10) / 6-31 G(d)$ level. CASPT2/CASSCF $(10,10) / 6-31 G(d)$ density differences of $\mathrm{S}_{1}$ and $\mathrm{T}_{1}$ states are given below.

\section{Conclusions}

Motivated by the distinct emission properties of propeller-shaped tetraphenyl-thiophene (TPT) in comparison to its carbon and silicon analogues, we have systematically explored the excited state relaxation mechanisms of this weak AIEgen in the vacuum and the solid state. We examined the role of the crystal structure, intermolecular interactions and exciton couplings on nonradiative and radiative pathways of TPT taking into account singlet and triplet states.

Given the weak interactions between the slip-stacked monomers in the solid state, exciton couplings are very small $(0.001-0.015 \mathrm{eV})$ in comparison with the reorganisation energies $(0.5-0.7 \mathrm{eV})$. Thus, the excited state processes will be mainly localised on the independent molecules. Reorganisation energies of large amplitude motions (low frequencies) are hampered in the solid state, however, the mode associated with the main nonradiative mechanism, the C-S stretching, is not significantly affected in the solid state. To understand the 
excited state mechanism, we analysed the excited state potential energy surfaces including crossings between geometries on the grounds of the RACI model.

In contrast with other propeller-shaped molecules where ring puckering coordinate drives the nonradiative mechanism, for TPT the C-S ring-opening pathway is the main reaction coordinate. The mechanism involves the population of two $\mathrm{S}_{1}$ minima: $\pi \pi^{*}$ to $\pi \sigma^{*}$. The transition from $\pi \pi^{*}$ to $\pi \sigma^{*}$ happens along the C-S coordinate via a crossing geometry. SOCs increase with the C-S distance, enhancing the population of triplet states. In the gas phase, both internal conversion and intersystem crossing are active and the $\mathrm{S}_{0} / \mathrm{S}_{1} \mathrm{MECI}$ and $\mathrm{X}\left(\mathrm{S}_{0} / \mathrm{T}_{1}\right)$ geometries are classically accessible, resulting in the quenching of emission. In the solid state, the $\mathrm{S}_{0} / \mathrm{S}_{1}$ MECI is hindered, while the $\mathrm{X}\left(\mathrm{S}_{0} / \mathrm{T}_{1}\right)$ remains accessible.

This mechanism explains why TPT is a weak AIEgen in comparison with other members of the propeller-shaped family. This represents the first investigation of the role of triplet states in the AIE mechanism of propeller-shaped molecules. Our calculations show that for effective enhancement of solid state luminescence, the population of triplet states should be also hampered. This could be achieved by imposing further constrains to the structure to restrict bond breaking and minimise spin-orbit couplings.

\section{Acknowledgement}

This research has been supported by the EPSRC (EP/R029385/1) and Leverhulme Trust (RPG-2019-122). We utilised Queen Mary's Apocrita HPC facility, supported by QMUL Research-IT and the ARCHER UK National Supercomputing Service (EP/L000202/1) via the Materials Chemistry Consortium and the Molecular Modelling Hub for computational resources, MMM Hub, which is partially funded by EPSRC (EP/P020194/1). We acknowledge the support from the School of Biological and Chemical Sciences at the Queen Mary University of London. 


\section{Supporting Information Available}

The following files are available free of charge.

- Supporting Information.

\section{References}

(1) Mei, J.; Leung, N. L. C.; Kwok, R. T. K.; Lam, J. W. Y.; Tang, B. Z. Aggregationinduced emission: together we shine, united we soar! Chem. Rev. 2015, 115, 1171811940.

(2) Shi, J.; Aguilar Suarez, L. E.; Yoon, S.-J.; Varghese, S.; Serpa, C.; Park, S. Y.; Lüer, L.; Roca-Sanjuán, D.; Milián-Medina, B.; Gierschner, J. Solid State Luminescence Enhancement in $\pi$-Conjugated Materials: Unraveling the Mechanism beyond the Framework of AIE/AIEE. The Journal of Physical Chemistry C 2017, 121, 23166-23183.

(3) Yin, S.; Peng, Q.; Shuai, Z.; Fang, W.; Wang, Y. H.; Luo, Y. Aggregation-enhanced luminescence and vibronic coupling of silole molecules from first principles. Physical Review B - Condensed Matter and Materials Physics 2006,

(4) Niu, Y.; Peng, Q.; Shuai, Z. Promoting-mode free formalism for excited state radiationless decay process with Duschinsky rotation effect. Science in China, Series B: Chemistry 2008, 51, 1153-1158.

(5) Crespo-Otero, R.; Li, Q.; Blancafort, L. Exploring Potential Energy Surfaces for Aggregation-Induced Emission-From Solution to Crystal. Chemistry - An Asian Journal 2019, 14, 700-714.

(6) Li, Q.; Blancafort, L. A conical intersection model to explain aggregation induced emission in diphenyl dibenzofulvene. Chemical Communications 2013, 49, 5966. 
(7) Peng, X.-L.; Ruiz-Barragan, S.; Li, Z.-S.; Li, Q.-S.; Blancafort, L. Restricted Access to a Conical Intersection to Explain Aggregation Induced Emission in Dimethyl Tetraphenylsilole. J. Mater. Chem. C 2016, 4, 2802-2810.

(8) Luo, J.; Xie, Z.; Lam, J. W. Y.; Cheng, L.; Chen, H.; Qiu, C.; Kwok, H. S.; Zhan, X.; Liu, Y.; Zhu, D.; Tang, B. Z. Aggregation-induced emission of 1-methyl-1,2,3,4,5pentaphenylsilole. Chem. Commun. 2001, 1740-1741.

(9) Hong, Y.; Lam, J. W. Y.; Tang, B. Z. Aggregation-induced emission. Chemical Society Reviews 2011, 40, 5361 .

(10) Stojanović, L.; Crespo-Otero, R. Understanding Aggregation Induced Emission in a Propeller-Shaped Blue Emitter. ChemPhotoChem 2019, 3, 907-915.

(11) Shen, P.; Zhuang, Z.; Zhao, Z.; Tang, B. Z. AIEgens based on main group heterocycles. J. Mater. Chem. C 2018, 6, 11835-11852.

(12) Nie, H.; Hu, K.; Cai, Y.; Peng, Q.; Zhao, Z.; Hu, R.; Chen, J.; Su, S.-J.; Qin, A.; Tang, B. Z. Tetraphenylfuran: aggregation-induced emission or aggregation-caused quenching? Mater. Chem. Front. 2017, 1, 1125-1129.

(13) Gu, Y.; Li, N.; Shao, G.; Wang, K.; Zou, B. Mechanism of Different Piezoresponsive Luminescence of 2,3,4,5-Tetraphenylthiophene and 2,3,4,5-Tetraphenylfuran: A Strategy for Designing Pressure-Induced Emission Enhancement Materials. The Journal of Physical Chemistry Letters 0, 0, 678-682.

(14) Zhao, Z.; Nie, H.; Ge, C.; Cai, Y.; Xiong, Y.; Qi, J.; Wu, W.; Kwok, R. T. K.; Gao, X.; Qin, A.; Lam, J. W. Y.; Tang, B. Z. Furan Is Superior to Thiophene: A Furan-Cored AIEgen with Remarkable Chromism and OLED Performance. Advanced Science 2017, 4, 1700005. 
(15) Guo, L.-X.; Xing, Y.-B.; Wang, M.; Sun, Y.; Zhang, X.-Q.; Lin, B.-P.; Yang, H. Luminescent liquid crystals bearing an aggregation-induced emission active tetraphenylthiophene fluorophore. J. Mater. Chem. C 2019, 7, 4828-4837.

(16) Yang, D.-J.; Lin, L.-Y.; Huang, P.-C.; Gao, J.-Y.; Hong, J.-L. Tetraphenylthiopheneterminated poly(acrylic acid) as pH- and bio-sensors by the aggregation-induced emission property. Reactive and Functional Polymers 2016, 108, 47 - 53, Current progress in advanced polymer materials for electronics/photonics functions.

(17) Lai, Y.-W.; Kuo, S.-W.; Hong, J.-L. Complexing AIEE-active tetraphenylthiophene fluorophore to poly(N-isopropyl acrylamide): fluorescence responses toward acid, base and metal ions. RSC Adv. 2012, 2, 8194-8200.

(18) Geng, T.; Zhang, W.; Zhu, Z.; Chen, G.; Ma, L.; Ye, S.; Niu, Q. A covalent triazinebased framework from tetraphenylthiophene and 2,4,6-trichloro-1,3,5-triazine motifs for sensing o-nitrophenol and effective I2 uptake. Polym. Chem. 2018, 9, 777-784.

(19) Christiansen, O.; Koch, H.; Jørgensen, P. The second-order approximate coupled cluster singles and doubles model CC2. Chemical Physics Letters 1995, 243, 409-418.

(20) Hättig, C.; Köhn, A. Transition moments and excited-state first-order properties in the coupled-cluster model CC2 using the resolution-of-the-identity approximation. Journal of Chemical Physics 2002, 117, 6939-6951.

(21) Hättig, C. Geometry optimizations with the coupled-cluster model CC2 using the resolution-of-the-identity approximation. Journal of Chemical Physics 2003, 118, $7751-7761$.

(22) Köhn, A.; Hättig, C. Analytic gradients for excited states in the coupled-cluster model CC2 employing the resolution-of-the-identity approximation. Journal of Chemical Physics 2003, 119, 5021-5036. 
(23) Roos, B. O.; Sadlej, A. J.; Malmqvist, P. A.; Andersson, K.; Wolinski, K. Secondorder perturbation theory with a CASSCF reference function. The Journal of Physical Chemistry 1990, 94, 5483-5488.

(24) Andersson, K.; Malmqvist, P. A.; Roos, B. O. Second-order perturbation theory with a complete active space self-consistent field reference function. The Journal of Chemical Physics 1992, 96, 1218-1226.

(25) Vancoillie, S.; Delcey, M. G.; Lindh, R.; Vysotskiy, V.; Malmqvist, P.-Å.; Veryazov, V. Parallelization of a multiconfigurational perturbation theory. Journal of Computational Chemistry 2013, 34, 1937-1948.

(26) Chai, J. D.; Head-Gordon, M. Long-range corrected hybrid density functionals with damped atom-atom dispersion corrections. Physical Chemistry Chemical Physics 2008, 10, 6615-6620.

(27) Bauernschmitt, R.; Ahlrichs, R. Treatment of electronic excitations within the adiabatic approximation of time dependent density functional theory. Chemical Physics Letters 1996, 256, 454-464.

(28) Casida, M. E.; Jamorski, C.; Casida, K. C.; Salahub, D. R. Molecular excitation energies to high-lying bound states from time-dependent density-functional response theory: Characterization and correction of the time-dependent local density approximation ionization threshold. Journal of Chemical Physics 1998, 108, 4439-4449.

(29) Stratmann, E. R.; Scuseria, G. E.; Frisch, M. J. An efficient implementation of timedependent density-functional theory for the calculation of excitation energies of large molecules. The Journal of Chemical Physics 1998, 109, 8218-8224.

(30) Caillie, C. V.; Amos, R. D. Geometric derivatives of excitation energies using SCF and DFT. Chemical Physics Letters 1999, 308, 249-255. 
(31) Furche, F.; Ahlrichs, R. Adiabatic time-dependent density functional methods for excited state properties. Journal of Chemical Physics 2002, 117, 7433-7447.

(32) TURBOMOLE V7.0 2015, a development of University of Karlsruhe and Forschungszentrum Karlsruhe GmbH, 1989-2007, TURBOMOLE GmbH, since 2007; available from http://www.turbomole.com.

(33) Roos, B. O.; Taylor, P. R.; Siegbahn, P. E. M. A complete active space SCF method (CASSCF) using a density matrix formulated super-CI approach. Chemical Physics 1980, 48, 157-173.

(34) Forsberg, N.; Malmqvist, P. A. Multiconfiguration perturbation theory with imaginary level shift. Chemical Physics Letters 1997, 274, 196-204.

(35) Maeda, S.; Ohno, K.; Morokuma, K. Updated Branching Plane for Finding Conical Intersections without Coupling Derivative Vectors. Journal of Chemical Theory and Computation 2010, 6, 1538-1545.

(36) Giannozzi, P. et al. QUANTUM ESPRESSO: A modular and open-source software project for quantum simulations of materials. Journal of Physics Condensed Matter 2009, 21, 395-502.

(37) Aragó, J.; Troisi, A. Dynamics of the excitonic coupling in organic crystals. Physical Review Letters 2015, 114, 1-5.

(38) Dommett, M.; Rivera, M.; Crespo-Otero, R. How Inter- and Intramolecular Processes Dictate Aggregation-Induced Emission in Crystals Undergoing Excited-State Proton Transfer. J. Phys. Chem. Lett. 2017, 8, 6148-6153.

(39) Rivera, M.; Dommett, M.; Crespo-Otero, R. ONIOM(QM:QM') Electrostatic Embedding Schemes for Photochemistry in Molecular Crystals. J. Chem. Theory Comput. 2019, 15, 2504-2516, PMID: 30865452. 
(40) Dapprich, S.; Komaromi, I.; Suzie Byun, K.; Morokuma, K.; Frisch, M. J. A new ONIOM implementation in Gaussian98. Part I. The calculation of energies, gradients, vibrational frequencies and electric field derivatives. Journal of Molecular Structure Theochem 1999, 461-462, 121.

(41) Chung, L. W.; Sameera, W. M.; Ramozzi, R.; Page, A. J.; Hatanaka, M.; Petrova, G. P.; Harris, T. V.; Li, X.; Ke, Z.; Liu, F.; Li, H. B.; Ding, L.; Morokuma, K. The ONIOM Method and Its Applications. Chemical Reviews 2015, 115, 5678-5796.

(42) Frisch, M. J. et al. Gaussian16 Revision A.03. 2016; Gaussian Inc. Wallingford CT.

(43) Merz, K. M.; Cornell, W. D.; Kollman, P. A.; Ferguson, D. M.; Cieplak, P.; Caldwell, J. W.; Bayly, C. I.; Gould, I. R.; Spellmeyer, D. C.; Fox, T. A Second Generation Force Field for the Simulation of Proteins, Nucleic Acids, and Organic Molecules. Journal of the American Chemical Society 2005, 117, 5179-5197.

(44) Aquilante, F. et al. Molcas 8: New capabilities for multiconfigurational quantum chemical calculations across the periodic table. Journal of Computational Chemistry 2016, $37,506-541$.

(45) Åke Malmqvist, P.; Roos, B. O.; Schimmelpfennig, B. The restricted active space (RAS) state interaction approach with spin-orbit coupling. Chemical Physics Letters 2002, $35 \%, 230-240$.

(46) Schimmelpfennig, B, Amfi, an atomic mean-field spin-orbit integral program. Computer code, 1996. University of Stockholm.

(47) Reimers, J. R. A practical method for the use of curvilinear coordinates in calculations of normal-mode-projected displacements and duschinsky rotation matrices for large molecules. Journal of Chemical Physics 2001, 115, 9103-9109. 
(48) Lai, C.-T.; Hong, J.-L. Aggregation-Induced Emission in Tetraphenylthiophene-Derived Organic Molecules and Vinyl Polymer. The Journal of Physical Chemistry B 2010, 114, $10302-10310$.

(49) Kölle, P.; Schnappinger, T.; de Vivie-Riedle, R. Deactivation pathways of thiophene and oligothiophenes: internal conversion versus intersystem crossing. Phys. Chem. Chem. Phys. 2016, 18, 7903-7915.

(50) Stenrup, M. Theoretical study of the radiationless deactivation mechanisms of photoexcited thiophene. Chemical Physics 2012, 397, 18 - 25.

(51) Salzmann, S.; Kleinschmidt, M.; Tatchen, J.; Weinkauf, R.; Marian, C. M. Excited states of thiophene: ring opening as deactivation mechanism. Phys. Chem. Chem. Phys. 2008, 10, 380-392.

(52) Prlj, A.; Curchod, B. F. E.; Corminboeuf, C. Excited state dynamics of thiophene and bithiophene: new insights into theoretically challenging systems. Phys. Chem. Chem. Phys. 2015, 17, 14719-14730.

(53) Schnappinger, T.; Kölle, P.; Marazzi, M.; Monari, A.; González, L.; de Vivie-Riedle, R. $\mathrm{Ab}$ initio molecular dynamics of thiophene: the interplay of internal conversion and intersystem crossing. Phys. Chem. Chem. Phys. 2017, 19, 25662-25670.

(54) Fazzi, D.; Barbatti, M.; Thiel, W. Modeling ultrafast exciton deactivation in oligothiophenes via nonadiabatic dynamics. Phys. Chem. Chem. Phys. 2015, 17, 7787-7799.

(55) Batignani, G.; Pontecorvo, E.; Ferrante, C.; Aschi, M.; Elles, C. G.; Scopigno, T. Visualizing Excited-State Dynamics of a Diaryl Thiophene: Femtosecond Stimulated Raman Scattering as a Probe of Conjugated Molecules. The Journal of Physical Chemistry Letters 2016, 7, 2981-2988, PMID: 27428853. 